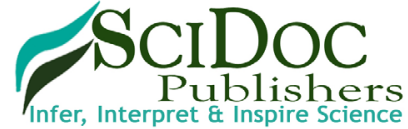

International Journal of Reproduction, Fertility \& Sexual Health (IJRFSH) ISSN: $2377-1887$

\title{
Ulipristal Acetate Effectively Medically Manages Symptomatic Leiomyomatous Uteri
}

Research Article

Nwanodi $\mathrm{OB}^{*}$

Locum Tenens Obstetrician \& Gynecologist, Salinas, CA, USA.

\section{Abstract}

Background: Symptomatic leiomyomatous uteri affect about 30\% of women. Associated anemia, dysmenorrhea, dyspareunia, heavy menstrual bleeding, and mass effects cause quality of life decrements. The 5 -year levonorgestrel intrauterine device (5yr-IUD) has been a mainstay of medical management for symptomatic leiomyomata. If approved, Ulipristal acetate (UPA), which entered the European and Canadian markets in 2012 and 2013, could change medical management of symptomatic leiomyomata in the United States.

Methods: PubMed search for the term "ulipristal acetate treatment fibroids" yielded five included articles. Hand search provided nine additional articles.

Results: The 5yr-IUD achieves maximal amenorrhea in $70 \%$ of users at 3 months. Dysmenorrhea improves in $84 \%$ of $5 \mathrm{yr}-$ IUD users. A Phase III trial with 451 participants compared UPA 5 and $10 \mathrm{mg}$ over four 12 -week treatment cycles. At the study end, menstruation was controlled in up to $77.5 \%$ of participants, with $70 \%$ or more participants in each group achieving amenorrhea. Combined median leiomyoma volume reduction for the three largest leiomyomata reached $67.4 \%$. Median health related QOL scores decreased from 56.9 and 55.2 respectively to 20.7 and 15.5 respectively after the fourth treatment cycle. Conclusion: UPA offers onset amenorrhea within 6 days irrespective of dose, whereas the 5yr-IUD may take 3-months to achieve amenorrhea. UPA decreases leiomyomata volume up to $65 \%$ within 1 -year, but, the 5yr-IUD does not reduce leiomyomata volume. The 5yr-IUD provides simultaneous contraception, whereas UPA requires separate contraception. As there are no significant benefits to higher dose treatment, UPA $5 \mathrm{mg}$ should have a place in gynecologic clinical practice.

Keywords: Amenorrhea; Dysmenorrhea; Heavy Menstrual Bleeding; Leiomyomatous Uterus; Levonorgestrel Intrauterine Device; Leiomyomata Mass Effect; Leiomyoma Size; Leiomyomata Volume; Selective Progesterone-Receptor Modulators; Ulipristal Acetate.

\section{Introduction}

Symptomatic leiomyomatous uteri affect about $30 \%$ of women [1]. In the United States, more than $80 \%$ of African American and $70 \%$ of Caucasian women have leiomyoma by age 50 , with an overall incidence of 12.8 per 1,000 woman years [1]. Symptomatic severity disparities are more pronounced than leiomyoma incidence disparities. African Americans women have larger and more numerous leiomyomata occurring up to 15 years earlier than do Caucasian women [1]. Heavy menstrual bleeding (HMB) and/ or mass symptoms affect about 20 to $40 \%$ respectively of women with leiomyomatous uteri [1]. Anemia, dysmenorrhea, dyspareunia, non-cyclic pelvic pain, and bladder tenesmus contribute to $53.7 \%$ of women with leiomyomata perceiving quality of life decrements [2]. Leiomyomata cost the United States up to USD 34 billion annually $[1,3]$. While hysterectomy is definitive treatment of symptomatic leiomyomatous uteri, hysterectomy is not the best option for all women, nor will immediate hysterectomy be a guaranteed insurance covered procedure. Therefore, medical management is important.

Since the levonorgestrel intrauterine system (5yr-IUD) became the only United States Food and Drug Administration (FDA) approved medication for HMB treatment in 2009, the 5yr-IUD may no longer be the most effective choice for HMB control [3]. The $5 \mathrm{yr}-\mathrm{IUD}$ achieves amenorrhea in $70 \%$ of users at 3 months, and in $8.8 \%$ to $63 \%$ of users at 12 months [4-6]. At 12 months $79 \%$ to $96 \%$ of 5 yr-IUD users are adherent $[4,5]$. Dysmenorrhea, im-

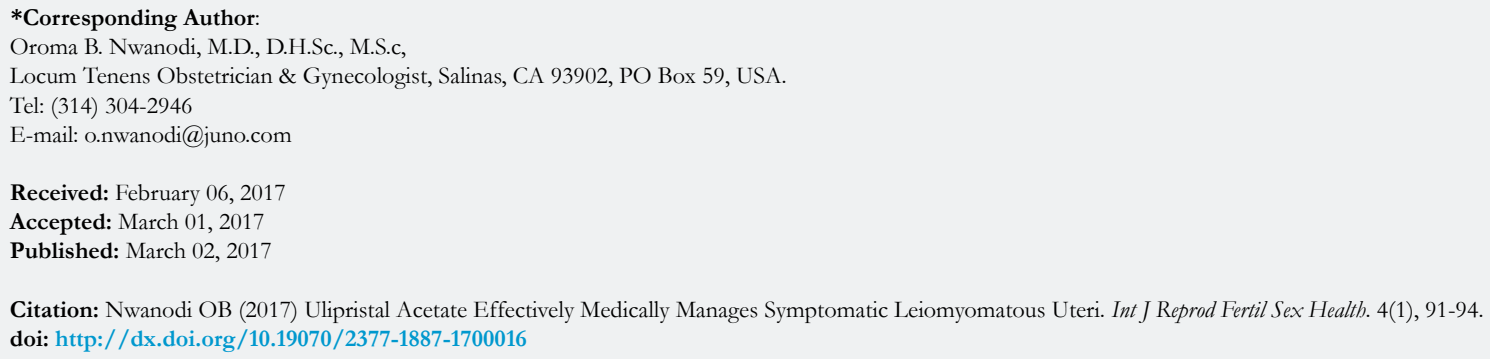

Copyright: Nwanodi $\mathbf{O B}^{\circ}$ 2017. This is an open-access article distributed under the terms of the Creative Commons Attribution License, which permits unrestricted use, distribution and reproduction in any medium, provided the original author and source are credited. 
proves in $84 \%$ of $5 y r-I U D$ users [6]. Despite increased expulsion from distorted uterine cavities, 5yr-IUD reinsertion is frequently requested [3]. However, the 5yr-IUD does not achieve leiomyoma size reduction. Also, the hormonal contraceptive benefits of the 5yr-IUD maybe exactly what some women do not want, instead preferring non-contraceptive, non-invasive medical management. Gonadotrophin-releasing hormone agonists (GnRHa) may be recommended for short-term symptomatic leiomyomatous uterus treatment [7]. However, GnRHa are known to cause hot flashes, which are most common in African American women [7, 8]. Thus, GnRHa serve to aggravate symptomatic severity disparities.

There is a non-contraceptive, non-invasive treatment of leiomyomatous uteri that promises to be more effective than the most effective invasive hormonal treatment option. Ulipristal acetate (UPA), a synthetic progesterone agonist/antagonist, also described as a selective progesterone-receptor modulator (SPRM), derived from 19-norprogesterone, dosed as $10 \mathrm{mg}$ by mouth daily for repeated 12 week courses has completed phase III trials in the United States $[3,9,10]$. UPA $5 \mathrm{mg}$ was approved for 3-months preoperative treatment of symptomatic leiomyomatous uteri in Europe in 2012 [7]. In 2014, UPA $5 \mathrm{mg}$ was approved for two 3 -month treatment courses in Europe [7]. UPA received initial Canadian approval in 2013, followed by approval for long-term intermittent use in 2016 [11]. Patients as described above present a clinical intervention, patient population, intervention, control, outcome, and treatment time (PICOT) question that can now be answered: In reproductive age women with symptomatic leiomyomatous uterus - dysmenorrhea and/or heavy menstrual bleeding (HMB) - will UPA dosed at 5 or $10 \mathrm{mg}$ by mouth daily for multiples of 12 week periods in comparison to the 5yr-IUD, achieve equivalent or better amenorrhea, adherence, improvement in dysmenorrhea, and leiomyoma size reduction?

\section{Methods}

The PubMed database was searched on February 1, 2017, search terms "ulipristal acetate treatment fibroids," with parameters free full text, human subjects, resulting in 10 articles. An in vitro fertilization case report, an ultrasonographical study, an endocrinology study, and two early trials were considered extraneous or redundant and excluded, leaving five articles, as shown in Figure 1. Hand search was performed for relevant background articles on symptomatic leimyomatous uteri, the 5yr-IUD, and ulipristal acetate, yielding nine articles, for a total of 14 included articles.

\section{The Data on Ulipristal Acetate}

Ulipristal is unique among SPRMs: Ulipristal has six mechanisms of action, whereas mifepristone has two, and asoprisnil and CP8947 each have four mechanisms of action [12]. Ulipristal induces caspase- 3 and $\mathrm{Bcl}-2$ dependent apoptosis, is anti-angiogenic and anti-proliferative, inhibits ovulation, reduces collagen deposition by matrix metalloproteinases (MMPs) and tissue inhibitors of metalloproteinases (TIMPs) modulation, and modulates estrogen receptors via mitochondrial and tumor necrosis factor (TNF) related apoptosis inducing ligand activation $[2,12]$. Multiple mechanisms of action sustain leiomyoma volume reduction for up to 6-months posttreatment facilitating intermittent treatment cycles [9]. Unlike GnRH agonists, UPA does not suppress estradiol to postmenopausal levels, so UPA is not associated with hot flashes $[2,9]$.

As UPA neither inhibits nor induces cytochrome P450 (CYP) 3A4, by which it is metabolized, UPA should not instigate drugdrug interactions [7]. Consistent with this, the commonest adverse effects with UPA are breast tenderness and headache that occur at a comparable rate to placebo [7]. However, UPA is affected by CYP34A inhibitors including ketoconazole and erythromycin, which will increase UPA levels, and by CYP34A inducers such as rifampicin and carbamazepine that will reduce UPA levels [7]. Iron supplementation does not significantly affect UPA bioavailability [7]. Oral bioavailability that is unaffected by food ingestion and a 35- to 43-hour half-life permit once daily oral dosing [7].

A mixed 12-week, open-label, and a 36-week double-blind, placebo controlled phase III trial of $10 \mathrm{mg}$ UPA enrolled 448 participants [13]. Each 12-week course of UPA was separated by two menstruations, with or without supplemental progestin. As this was a phase III trial, the initial open-label, format is valid. The study used sample sizes sufficient to achieve $95 \%$ confidence intervals (CI) within $+/-6 \%$ [13]. Wilson scores, Kaplan Meier probability estimates, and Wilcoxon rank sum test were performed. After 12 weeks of UPA, $78.5 \%$ of participants were amenorrheic $(95 \%$ CI, $72.3 \%-83.5 \%$ ). At 6 and 12 months, $88.5 \%$ and $89.7 \%$ of participants were amenorrheic [13]. At 3-months the median vol-

Figure 1. Article Inclusion Process.

Studies identified through 2 search strategies stated in the Methods.

Total $\mathrm{n}=19$

1. PubMed search on February 1, 2017, search terms "uliptistal acetate treatment fibroids," parameters human subjects, English language, free full text $(\mathrm{n}=10)$.

2. Hand Search on February 1, 2017, $(n=9)$. 
ume reduction of the largest three leiomyomata was $45.1 \%$, at 12 months $72.1 \%$, and at 3 months off UPA $58.8 \%$. Quality of life improved, approximating to that of women who had surgical treatment. Pain and discomfort decreased [13].

Adherence in the open-label trial was $63 \%$, which was affected by the loss of patients who had already planned to undergo surgical treatment after the initial 12 weeks UPA treatment. In clinical practice, patients may receive preoperative medical management to shrink leiomyomata and build iron stores, therefore, these patients represent real clinical practice. Adherence in the double-blind phase was $81 \%$ [13]. UPA dosed at $10 \mathrm{mg}$ by mouth daily for 12 week intervals separated by 2 menstruations achieves amenorrhea in a greater proportion of women than the $5 \mathrm{yr}-\mathrm{IUD}$ at 3, 6, and 12 months of treatment $(78.5 \%$ and $89.7 \%$ versus $70 \%$ and $8.8 \%$ to $63 \%$, respectively). When participant losses for previously planned surgical treatment are excluded, UPA achieves equivalent adherence to the $5 \mathrm{yr}-\mathrm{IUD}$ ( $81 \%$ versus $79 \%$ to $96 \%$ ). Reduction in dysmenorrhea by UPA was about $45 \%$, contrasting to the $5 \mathrm{yr}-\mathrm{IUD}$ at $84 \%$ [13].

The study performance and instruments appear to be consistent with good clinical research. Outcomes were defined in keeping with the literature: Amenorrhea, menstrual blood loss by Pictorial Blood-Loss Assessment Chart (PBAC), and ultrasound measurement of fibroid size [13]. Secondary pain and quality of life endpoints were evaluated via validated questionnaires: The ShortForm McGill Pain questionnaire, the EQ-5D questionnaire, and the Uterine Fibroid Symptom and Health-Related Quality of Life questionnaire. Consistent with phase III trials any adverse events were evaluated [13]. UPA is unlikely to have more harms than existing hormonal and non-hormonal treatments for HMB and dysmenorrhea. The study has several biases. Due to the initial open-label portion, investigator outcome assessment bias was possible [14]. Objective outcomes assessment, adjudication committees, and procedural decision pathways can reduce investigator outcome assessment bias [14]. Only objective outcomes assessment was used, reducing investigator outcome assessment bias [13]. However, selection bias occurred as some patients specifically selected UPA as a pre-surgical treatment. Once these patients were excluded in the second phase, losses to follow-up or UPA discontinuations were equivalent to data for the 5yr-IUD [13].

The international Phase III trial of two 12-week treatment cycles of UPA 5 and $10 \mathrm{mg}$, enrolled 451 premenopausal women aged 18 to 50 years, body mass index (BMI) of 18 to $40 \mathrm{~kg} /$ $\mathrm{m}^{2}$, with symptomatic leiomyomata, sonographically sized from 3 to $12 \mathrm{~cm}$ diameter [9]. At the end of each treatment cycle menstruation measured by PBAC was controlled in more than $80 \%$ of participants irrespective of UPA dose. At the end of the second treatment cycle combined median leiomyoma volume reduction for the three largest leiomyomata was $54 \%$ and $58 \%$ respectively [9]. Median visual analogue scale pain scores reduced to 6 and 5 respectively at the end of the second treatment cycle [9]. There were 21 treatment discontinuations for adverse events, with only four instances of HMB and a partial leiomyoma expulsion being related to UPA [9]. Three participants in the $5 \mathrm{mg}$ group and five patients in the $10 \mathrm{mg}$ group had surgical interventions. Endometrial pathology did not progress during the trial [9].

An additional International Phase III trial compared UPA 5 and $10 \mathrm{mg}$ over four 12-week treatment cycles with 451 participants as described above [10]. At the end of the fourth treatment cycle menstruation measured by PBAC was controlled in $77.5 \%$ and $76 \%$ of participants respectively [10]. At least $70 \%$ of participants in each group achieved amenorrhea, on average occurring within 1 -week of study initiation [10]. At the end of the fourth treatment cycle combined median leiomyoma volume reduction for the three largest leiomyomata was $65 \%$ and $67.4 \%$ respectively, $\mathrm{p}$ $>.08$ [9]. Median visual analogue scale pain scores were reduced to 5 and 7 respectively during treatment [9]. Median health related QOL scores decreased from 56.9 and 55.2 respectively to 20.7 and 15.5 respectively after the fourth treatment cycle [10]. Reported UPA related adverse events decreased with each cycle from 47 to 11 for UPA $5 \mathrm{mg}$ and from 43 to 14 for UPA $10 \mathrm{mg}$ [10]. Seven participants in the $5 \mathrm{mg}$ and nine participants in the $10 \mathrm{mg}$ group had surgery, only three of which were previously planned procedures. All surgeries were related to symptomatic leiomyomata [10]. There were 21 UPA related adverse effect treatment discontinuations [10]. UPA related adverse effects included five cases of menorrhagia, two spontaneous leiomyoma expulsions, one partial leiomyoma expulsion, one necrotic leiomyoma, one new diagnosis each of abdominal pain, bipolar disorder, endometriosis, and lumbago [10]. Again, endometrial pathology did not progress during the trial [10].

Compared to the 5yr-IUD, UPA offers quicker onset amenorrhea (within 6 days without a dose dependent effect versus 3-months), and decreased leiomyomata volume $(65 \%$ within 1 -year versus no volume reduction). Whereas the $5 \mathrm{yr}-\mathrm{IUD}$ entails a risk of uterine perforation requiring surgical intervention, UPA is non-invasive. However, the 5yr-IUD provides simultaneous contraception, whereas UPA requires separate contraception. As there are no significant outcomes benefits to UPA $10 \mathrm{mg}$ over UPA $5 \mathrm{mg}$, in clinical practice UPA $5 \mathrm{mg}$ should be adequate.

\section{Conclusion}

This PICOT question answers a clinical question gynecologists are confronted with on a weekly basis: In reproductive age women with symptomatic leiomyomatous uterus - dysmenorrhea and/ or heavy menstrual bleeding (HMB) - will UPA dosed at 5 or $10 \mathrm{mg}$ by mouth daily for multiples of 12 week periods in comparison to the 5yr-IUD, achieve equivalent or better amenorrhea, adherence, improvement in dysmenorrhea, and leiomyoma size reduction? For instance, a pre-menopausal woman who has just come off the vaginal contraceptive ring after several years' use, has increasingly heavy menstruation, leading to a finding of a submucosal leiomyoma. She does not want to start another hormonal conceptive and is unsure about surgical treatment. As the data on UPA seems better than the 5yr-IUD, and as $10 \mathrm{mg}$ UPA is not significantly more effective than $5 \mathrm{mg}$ UPA, she chooses a trial of $5 \mathrm{mg}$ UPA. Hopefully, treatment of HMB due to leiomyomata will no longer be off-label use of UPA in the United States (but an approved use in Europe). A double blind, randomized control trial to directly compare UPA and the 5yr-IUD may never be undertaken as an inert IUD is not biologically plausible: A synthetic diffusing membrane IUD (without active hormone) in the uterus will still exert size and shape physical mass effects on the endometrium and uterine volume. Providers for women with HMB seeking non-hormonal treatment in the United States should be interested in the progress of UPA through United States Food and Drug Administration approval and potential price reductions. 


\section{Acknowledgement}

This mini review is based on coursework previously presented to the College of Graduate Health Studies in partial fulfillment of the requirements for the Doctor of Health Sciences Degree A.T. Still University.

\section{References}

[1]. Styer AK, Rueda BR (2016) The epidemiology and genetics of uterine leiomyoma. Best Prac Res Clin Obstet Gynaecol. 34: 3-12.

[2]. Biglia N, Carinelli S, Maiorana A, D'Alonzo M, Lo Monte G, et al., (2014) Ulipristal acetate: a novel pharmacological approach for the treatment of uterine fibroids. Drug Des Devel Ther. 8285-292.

[3]. Kashani NB, Centini G, Morelli SS, Weiss G, Petraglia F (2016) Role of medical management for uterine leiomyomas. Best Prac Res Clin Obstet Gynaecol. 34: 85-103.

[4]. Blitzer J, Heikinheimo O, Nelson AL, Calaf-Alsina J, Fraser IS (2015) Medical management of heavy menstrual bleeding: a comprehensive review of the literature. Obstet gynecol surv. 70(2): 115-130.

[5]. Jensen J, Mansour D, Lukkari-Lax E, Inki P, Burock K, et al., (2013) Bleeding patterns with the levonorgestrel-releasing intrauterine system when used for heavy menstrual bleeding in women without structural pelvic pathology: a pooled analysis of randomized controlled studies. Contraception. 87(1):
107-112.

[6]. Zalewski MM, Neulen J (2014) Update of Conservative Systemic Treatment of Uterine Fibroids. Curr Obstet Gynecol Rep. 3(3): 191-195.

[7]. Pohl O, Zobrist RH, Gotteland JP (2015) The clinical pharmacology and pharmacokinetics of ulipristal acetate for the treatment of uterine fibroids. Reprod Sci. 22(4): 476-483.

[8]. Thurston RC, Joffe H (2011) Vasomotor Symptoms and Menopause: Findings from the Study of Women's Health Across the Nation. Obstet Gynecol Clin North Am. 38(3): 489-501.

[9]. Donnez J, Hudecek R, Donnez O, Matule D, Arhendt HJ, et al., (2015) Efficacy and safety of repeated use of ulipristal acetate in uterine fibroids. Fertil Steril. 103(2): 519-527.e3.

[10]. Donnez J, Donnez O, Matule D, Ahrendt HJ, Hudecek R, et al., (2016) Long-term medical management of uterine fibroids with ulipristal acetate. Fertil Steril. 105(1): 165-173.e4.

[11]. De Francesco L (2016) Fibristal $^{\oplus}$, the first and only medication indicated for uterine fibroids, receives approval from Health Canada for long term intermittent treatment. PR Newswire.

[12]. Khan AT, Shehmar M, Gupta JK (2014) Uterine fibroids: current perspectives. Int J Women's Health. 6(2): 95-114.

[13]. Donnez J, Vázquez F, Tomaszewski J, Nouri K, Bouchard P, et al., (2014) Long-term treatment of uterine fibroids with ulipristal acetate. Fertil steril. 101(6): 1565-1573.

[14]. Kahan BC, Cro S, Doré CJ, Bratton DJ, Rehal S, et al., (2014) Reducing bias in open-label trials where blinded outcome assessment is not feasible: strategies from two randomised trials. Trials. 15: 456. 